\title{
CONFUSÃO SEXUAL PARA O CONTROLE DE GRAPHOLITA MOLESTA (LEPIDOPTERA: TORTRICIDAE), EM POMARES DE MACIEIRA, EM FRAIBURGO (SC), BRASIL $\left(^{\mathbf{1}}\right)$
}

\author{
LINO B. MONTEIRO ( $\left.{ }^{2}\right)$; ALEXANDRE DE SOUZA $\left({ }^{3}\right)$; LUIZ BELLI $\left({ }^{3}\right)$
}

\begin{abstract}
RESUMO
O controle de Grapholita molesta (Lepidoptera: Tortricidae) foi avaliado pela técnica de confusão sexual em macieira no município de Fraiburgo (SC). A formulação do feromônio sintético utilizada foi da marca Quant G.m. (400 mg do acetato de (Z/E) - 8 - dodecenila 14\% p/v), com 500 difusores por hectare, distribuídos em um pomar de macieira comercial 'Fuji' de 2,52 ha. O tratamento com confusão sexual foi comparado com a testemunha convencional. O monitoramento para moscas-das-frutas, Anastrepha fraterculus (Diptera: Tephritidae); mariposa oriental, Grapholita molesta e lagarta enroladeira, Bonagota cranaodes (Lepidoptera: Tortricidae) foi realizado para decidir se haveria necessidade de pulverização de inseticidas. Não houve capturas de G. molesta na armadilha do Tipo Delta, instalada no tratamento com confusão sexual até 93 dias após a liberação do feromônio. Nesse período, realizaram-se duas pulverizações para moscas-das-frutas e lagarta-enroladeira no tratamento com confusão sexual e oito pulverizações no tratamento convencional. Os danos causados por G. molesta em ambos os tratamentos não ultrapassaram $0,5 \%$, mesmo com a redução de $77 \%$ dos inseticidas no tratamento com confusão sexual. Verifica-se, portanto, que a técnica de confusão sexual é eficiente para o controle de G. molesta.
\end{abstract}

Palavras-chave: macieira, confundimento de macho, mariposa oriental, controle químico.

\section{ABSTRACT \\ MATING DISRUPTION FOR THE CONTROL OF Grapholita molesta (LEPIDOPTERA: TORTRICIDAE), IN FRAIBURGO, SANTA CATARINA, BRAZIL}

The control of Grapholita molesta (Lepidoptera: Tortricidae) was assessed by using the mating disruption technique in an apple orchard in Fraiburgo, SC. Quant G.m. synthetic pheromone formulation (400 mg of (Z/E) - 8 - dodecenyl acetate $14 \% \mathrm{p} / \mathrm{v}$ ) was used, with 500 dispensers per hectare, distributed over a 2.52 hectare 'Fuji' commercial orchard. The mating disruption treatment was compared with a chemical control treatment. The action threshold was determined by monitoring fruit flies Anastrepha fraterculus (Diptera: Tephritidae), G. molesta and leafroller Bonagota cranaodes (Lepidoptera: Tortricidae). G. molesta was caught in the Delta traps installed in the mating disruption plot until 93 days from release of the pheromone. In this period, the mating disruption treatment was sprayed twice for fruit flies and leafrollers, and the chemical control plot received eight pesticides application. The damage caused by $G$. molesta in both treatments did not exceed $0.5 \%$, even with a $77 \%$ reduction of insecticide in the mating disruption plot. We concluded that the mating disruption technique was efficient in the control of $G$. molesta.

Key words: apple orchard, mating disruption, oriental moth, chemical control.

$\left({ }^{1}\right)$ Recebido para publicação em 19 de abril de 2006 e aceito em 21 de julho de 2007.

$\left({ }^{2}\right)$ Departamento de Fitotecnica e Fitossanidade da Universidade Federal do Paraná, Caixa Postal 19061, 81831-990, Curitiba, PR. E-mail:lbmonteiro@terra.com.br $\left(^{*}\right)$ Autor correspondente.

$\left({ }^{3}\right)$ Agrícola Fraiburgo Ltda., Rod. SC 453, km 35, 89580-000 Fraiburgo (SC). 


\section{INTRODUÇÃO}

A macieira é cultivada nos pólos de Vacaria, no Rio Grande do Sul, e Fraiburgo e São Joaquim, em Santa Catarina, respondendo por $80 \%$ dos 32.981 ha de área plantada no Brasil (IBGE, 2005). A produção brasileira de maçãs é 700.000 toneladas e $80 \%$ é consumida no mercado brasileiro (Aquino e BENITEZ, 2005). A cultura da macieira caracteriza-se por necessitar de mão-de-obra abundante para a realização dos tratos culturais, empregando 23.500 e 6.800 pessoas, respectivamente, de modo permanente e temporário.

Os pomares de macieira de Fraiburgo caracterizam-se por grandes áreas cultivadas; na região estão localizadas quatro empresas, que são responsáveis por, aproximadamente, $25 \%$ da produção nacional, e juntas possuem 6.000 ha de pomar. Em função da proximidade dos pomares, há forte pressão de pragas, o que exige aplicações sucessivas de diferentes produtos químicos para seu controle. Entre as pragas afetadas, destaca-se o ácaro vermelho, Panonychus ulmi (Koch) (Acari: Tetranychidae), cujas populações aumentam quando há desequilíbrio ambiental (MONTEIRO, 1994), sobretudo, em decorrência do uso de inseticidas para o controle de Grapholita molesta $($ Busck $)(=$ Cydia molesta), Bonagota cranaodes Meyrick (Lepidoptera: Tortricidae) e Anastrepha fraterculus Wied (Diptera: Tephritidae).

A mariposa oriental, G. molesta, ataca a cultura da macieira em quase todas as áreas de plantio no Brasil (ORTH, et al., 1986). Em Fraiburgo, realizase a captura da mariposa em armadilha Delta todos os meses, inclusive no inverno. Seus ataques iniciamse em pessegueiro em meado de agosto e, após a colheita dos pêssegos, as flutuações concentram-se nas macieiras (HICKEL, 1993). A larva constrói galerias nos ponteiros dos ramos novos e, no fim de novembro, penetra nos frutos (GoNZALEZ, 2003).

Em razão da presença de pessegueiros, ameixeiras e pereiras na região de Fraiburgo a mariposa oriental e moscas-das-frutas tornaram-se pragas cada vez mais problemáticas nos últimos anos, o que requer aumento no uso de inseticidas, principalmente em 'Fuji', que é uma variedade de ciclo longo. Os resultados com os tratamentos-inseticidas têm sido contraditórios, às vezes totalmente ineficientes. Esse problema pode estar relacionado com resistência dos tortricídeos a alguns produtos no Brasil, como demonstraram BOUvier et al. (1995), SAUPHANOR E Bouvier (1995) e CHARMillot (1995) para Cydia pomonella L. (Lepidoptera: Tortricidae) na Europa, e/ou com a época de aplicação, visto que os inseticidass pulverizados para o controle de G. molesta são produtos organofosforados, e há somente um regulador de crescimento de inseto (ANDREI, 1999).

Nesse contexto, novas estratégias de controle estão sendo testadas para reduzir o uso de inseticidas, entre elas a confusão sexual (DEGEN et al., 2005). Esta técnica consiste em saturar o ambiente de um pomar com feromônios sexuais, a fim de desorientar o macho e impedir o acasalamento (Vilela e Lucia, 2001). As formulações de confusão sexual para G. molesta são utilizadas em pomares de pessegueiro e macieira em vários países (CHARMillot et al., 1997; Degen et al., 2005; Kovanci et al., 2005).

O objetivo deste estudo foi testar a técnica de confusão sexual de G. molesta em macieira e verificar sua viabilidade em associação ao manejo de outras pragas, nas condições de Fraiburgo (SC).

\section{MATERIAL E MÉTODOS}

O trabalho foi desenvolvido em um pomar de macieira 'Fuji' implantado em 1992 no espaçamento de $4,5 \mathrm{~m}$ entre linhas e $1,5 \mathrm{~m}$ entre plantas $(2.481$ macieiras), totalizando 2,52 hectares. O tratamento com confusão sexual estava a 50 metros de um pomar de macieira abandonado, limitando-se com campo aberto e uma parcela de macieira de mesma variedade e pertencente ao mesmo pomar de igual tamanho, constituindo-se o tratamento convencional (químico).

A formulação de confusão sexual utilizada foi a marca Quant G.m.® (BASF Española S.A., Barcelona), cujo ingrediente ativo (i.a.) do atrativo para G. molesta é o acetato de (Z/E) - 8 - dodecenila $14 \% \mathrm{p} / \mathrm{v}$. Empregaram-se difusores de ampolas duplas e somente em uma delas continha o feromônio. Cada ampola possui $400 \mathrm{mg}$ de i.a., sendo utilizados 500 difusores por hectare, com duração de 90 a 120 dias de liberação. Os difusores foram colocados nas macieiras à altura de 1,8 m em 7/12/1999, para que a liberação do feromônio pudesse agir no período de formação da maçã até próximo à colheita. A distribuição dos difusores foi feita a cada $4,5 \mathrm{~m}$ na entrelinha e $5 \mathrm{~m}$ na linha de plantio, totalizando 1.118 (444/ha), com 177,6 g i.a. ha ${ }^{-1}$; na bordadura da parcela, foi dobrado o número de difusores (142 difusores), com o objetivo de compensar a perda da concentração de feromônio devido à ação de ventos na periferia da parcela. Antes da instalação dos difusores foi realizada uma pulverização com clorpirifós (Lorsban 480, $120 \mathrm{~mL} / 100 \mathrm{~L}$ de calda) em ambas as parcelas para reduzir a população de grafolita, visto que a eficiência do confundimento de machos é reduzida em altas populações iniciais (Charmillot e PAsquier, 2001) 
A flutuação de G. molesta e B. cranaodes foi monitorada com uma armadilha do tipo Delta por praga e tratamento, cujas marcas dos feromônios foram, respectivamente, Biografolita ${ }^{\circledR}$, BioControle - São Paulo (SP), e Iscalure Bonagota ${ }^{\circledR}$, Isca Technologias - Ijuí (RS). Contou-se número de machos capturados duas vezes por semana, e os feromônios foram trocados a cada 45 dias, em ambas as parcelas. A. fraterculus foi monitorada com frascos caça-mosca do tipo McPhail, com suco de uva a $25 \%$, marca Milani ${ }^{\circledR}$, Wessanen do Brasil - Mogi das
Cruzes (SP), trocados a cada sete dias. Os tratamentos fitossanitários no tratamento convencional foram realizados com base nas decisões normalmente utilizadas pela maioria dos produtores da região de Fraiburgo, apoiada na relação entre capturas e o nível de controle (NC). O NC foi fixado em 20 machos/armadilha/ amostragem (G. molesta e B. cranaodes) e 0,5 capturas/frasco/dia (A. fraterculus). Os inseticidas utilizados para o controle de pragas no pomar analisado estão relacionados na Tabela 1.

Tabela 1. Inseticidas utilizados nos tratamentos com confusão sexual para o controle de G. molesta e convencional, em macieira 'Fuji', Fraiburgo, SC.

\begin{tabular}{lllll}
\hline \multirow{2}{*}{ Data de pulverização } & \multicolumn{2}{c}{ Tratamento } & Concentração $\left({ }^{1}\right)$ & Praga-alvo \\
\cline { 2 - 2 } & Confusão sexual & Convencional &
\end{tabular}

$24 / 9 / 1999$

$18 / 10 / 1999$

$8 / 11 / 1999$

24/11/1999

$7 / 12 / 1999\left(^{2}\right)$

22/12/1999

$3 / 1 / 2000$

$10 / 1 / 2000$

$19 / 1 / 2000$

$26 / 1 / 2000$

$18 / 2 / 2000$

$26 / 2 / 2000$

$4 / 3 / 2000$

$13 / 3 / 2000$

$3 / 4 / 2000$

Tebufenozide
Clorpirifós
Cimetoato
Clorpirifós
Clorpirifós
X
X
X

Phosmet

$\mathrm{X}$

Genitrothion $\mathrm{X}$ $X$

Clorpirifós

Dimetoato
Ttebufenozide

Clorpirifós

dimetoato

Clorpirifós

Clorpirifós

Methidathion

Fenitrothion

Fenitrothion

Clorpirifós

Dimetoato

Fenitrothion

Clorpirifós

Clorpirifós

Clorpirifós

Dimetoato

$\mathrm{ml} / 100\left(^{1}\right)$

\section{0}

150

150

150

150

100

150

150

150

150

150

150

150

150

150
Lagartas de solo

Lagartas de solo Aphis sp.

Lagartas de solo

Lagartas em geral

A. fraterculus, G. molesta

G. molesta, B. cranaodes

G. molesta

A. fraterculus / G. molesta $\left({ }^{3}\right)$

A. fraterculus

B. cranaodes

- $\left({ }^{4}\right)$

B. cranaodes

$-\left({ }^{4}\right)$

G. molesta, B. cranaodes $\left({ }^{5}\right)$

$\left({ }^{1}\right)$ Concentração do produto comercial. $\left({ }^{2}\right)$ Data de instalação dos difusores da confusão sexual; $\left({ }^{3}\right)$ A primeira praga relaciona-se com o tratamento com confusão sexual e a segunda com o convencional; $\left({ }^{4}\right)$ Pulverização realizada pelo produtor sem levar em consideração o monitoramento e $\left({ }^{5}\right)$ Pulverização realizada abaixo do nível de controle.

A avaliação do efeito dos feromônios sobre a comunicação de G. molesta foi realizada pelo cálculo do índice de interrupção do acasalamento (IIA), sendo IIA $=(C-T / C)^{*} 100$, em que C é a média de machos de G. molesta capturados por armadilha no tratamento com confusão sexual e $\mathrm{T}$, o número de capturas no tratamento convencional (Molinari et al., 2000).

A avaliação de danos em frutos foi realizada em $26 / 1 / 2000,15 / 2 / 00,2 / 3 / 00,17 / 3 / 00$ e $12 / 4 / 00$ (colheita), determinando-se a porcentagem de frutos com danos e localizados em um ramo submestre, situados no lado oeste e na parte mediana superior da planta, amostrados em 30 plantas marcadas por tratamento (CHARmillot et al., 1995). Na colheita, efetuou-se a avaliação em todos os frutos das plantas marcadas.
A avaliação dos danos anteriores à instalação dos difusores ocorreu em 2/12/1999, e foram anotados os danos de 12.000 frutos distribuídos em 15 macieiras de cada tratamento. Os danos foram observados para G. molesta, caracterizado por penetração de lagarta na maçã com orifício que continham dejetos, B. cranaodes, cujos sintomas são lesões na epiderme, normalmente verificadas próximo ao pedúnculo, e lagartas que habitam a vegetação rasteira, as quais provocam lesões superficiais na epiderme da maçã (as lagartas não foram identificadas). A porcentagem de danos de G. molesta, verificada no período anterior à instalação da confusão sexual, foi de $0,4 \%$ e $0,0 \%$ nos tratamentos, com confusão sexual e convencional respectivamente. 
Os danos de B. cranaodes nos dois tratamentos foram, respectivamente $1,4 \%$ e $0,4 \%$. Para análise estatística de danos, efetuou-se análise de variância comparando-se as médias pelo teste de Tukey, a 5\% de probabilidade.

\section{RESULTADOS E DISCUSSÃO}

As capturas de G. molesta no tratamento com confusão sexual foram menores em relação ao tratamento convencional (Figura 1). Entre 8/12/1999 e 10/3/2000 não houve capturas no tratamento com confusão sexual e o índice de interrupção de acasalamento (IIA) foi de $100 \%$. A partir de 10/3/2000, houve capturas de grafolita na armadilha instalada no tratamento com confusão sexual, provavelmente devido à redução da concentração do feromônio, após 93 dias de liberação, demonstrado pela diminuição do IIA para $18,6 \%$ nos últimos 32 dias do ciclo vegetativo da 'Fuji'. A confusão sexual não foi reaplicada nesses 32 dias até a colheita, visto que o custo benefício não compensava a aquisição do produto.

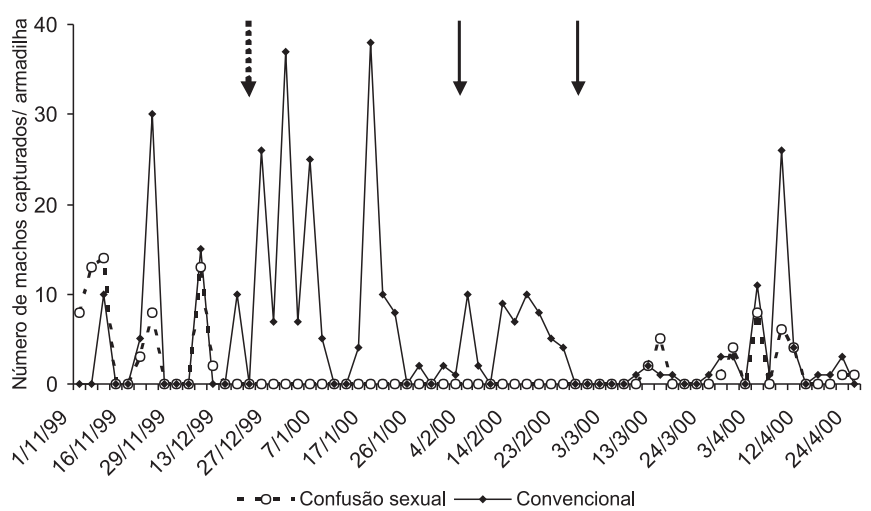

Figura 1. Flutuação populacional de machos de G. molesta no tratamento com confusão sexual para o controle de G. molesta e convencional, em pomar de macieira 'Fuji'. A seta tracejada indica a data de colocação dos difusores para a confusão sexual e as setas com linha cheia indicam as datas de pulverização com inseticidas no período de 93 dias de ação da confusão sexual, Fraiburgo (SC), 1999/2000.

No período compreendido entre a data de instalação dos difusores e 10/3/2000, realizaram-se duas pulverizações no tratamento com confusão sexual, motivados por moscas-das-frutas (19/1/2000) e $B$. cranaodes (18/2/2000). A última aplicação foi justificada, pois as capturas de mariposas estavam acima do NC desde 7/2/2000 (Figura 2). Essas pulverizações podem ter influenciado os resultados de danos provocados por G. molesta neste tratamento, entretanto, como não havia capturas na armadilha supõe-se que o desenvolvimento de lagartas foi nulo no tratamento. No tratamento convencional, foram realizadas oito pulverizações, das quais duas foram especificamente para o controle de moscas-das-frutas e com ação sobre G. molesta (22/12/1999 e 26/1/ $2000)$, três especificamente para $G$. molesta $(3 / 1,10 /$ 1 e 19/1) e três para $B$. cranaodes (3/1, 18/2 e 4/3/ 2000). Todas as pulverizações foram relacionadas com a flutuação de pragas, exceto a de 26/2/2000, que não teve nenhum critério firmado no NC (Tabela 1). Nesse período, foi possível reduzir em $77 \%$ as pulverizações com inseticidas no tratamento com confusão sexual.

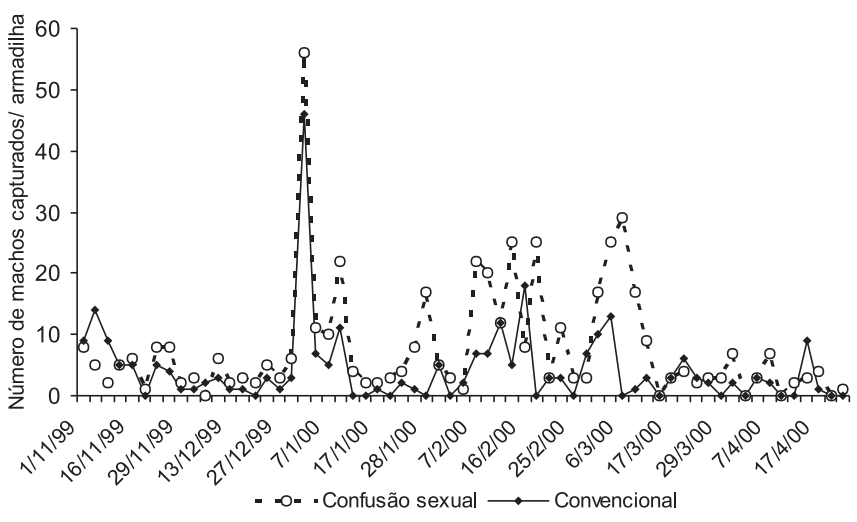

Figura 2. Flutuação populacional de machos de $B$. cranaodes no tratamento com confusão sexual para o controle de G. molesta e convencional em pomar de macieira 'Fuji', Fraiburgo (SC), 1999/2000.

Nos últimos 32 dias antes da colheita houve duas aplicações de inseticidas no tratamento com confusão sexual; em ambas as datas os níveis de capturas estavam abaixo do NC. Entretanto, a presença de G. molesta na armadilha Delta justificou as pulverizações, visto que o efeito de confusão entre machos e fêmeas estava reduzido e havia a possibilidade de acasalamento, pois o IIA era de $18,6 \%$. Além disso, a colheita leva em média 25 dias para ser concluída, tempo suficiente para que nova geração de lagartas ocasionasse danos às maçãs. $\mathrm{O}$ período de pré-colheita da 'Fuji' é suscetível ao dano de pragas, possivelmente devido à cor dos frutos e pelas substâncias liberadas por eles, o que têm motivado as aplicações de inseticidas (CHARMILLOT et. al., 1997, Bоттоn et al., 2005).

Não foi verificado nenhum fruto com danos de G. molesta em ambos os tratamentos até 17/3/2000, última avaliação antes da colheita (Tabela 2). Neste 
período de 100 dias, os danos ocorridos nos frutos foram provocados por lagartas que habitam a vegetação rasteira, que somados não ultrapassaram $0,55 \%$, e por B. cranaodes, os quais foram significativamente maiores no tratamento com confusão sexual do que no convencional, embora os valores médios sejam considerados baixos, 0,48 e $0,21 \%$ respectivamente. Mediante os resultados de danos das três pragas, observaram-se que as decisões de tratamento com confusão sexual foram adequadas, sendo a estratégia com feromônio, associada a três pulverizações com inseticidas, equivalente aos nove realizados no convencional.

As maiores porcentagens de danos foram verificadas na colheita de $12 / 4 / 2000$. G. molesta causou danos inferiores a $0,5 \%$ no tratamento com confusão sexual, sendo insignificantes economicamente (Tabela 2). O resultado é semelhante ao obtido por Kovanci et al. (2004). É possível que as duas aplicações em pré-colheita tenham colaborado para que os danos permanecessem baixos.

Os danos por B. cranaodes foram significativamente maiores no tratamento com confusão sexual quando comparados com o tratamento convencional (Tabela 2). Apesar disso, a diferença foi pequena $(1 \%)$ levando em consideração que foram realizadas oito pulverizações no tratamento convencional e apenas duas no tratamento com confusão sexual. É possível que o feromônio utilizado para o controle de G. molesta tenha interferido no acasalamento de mariposas de B. cranaodes. Em 1996, foram realizados testes de monitoramento com feromônio de $B$. cranaodes, isolados pela empresa japonesa Fuji Flavors, e houve capturas tanto machos de G. molesta como de B. cranaodes (Monteiro, não publicado).

Tabela 2. Danos (\%) de pragas em tratamento com confusão sexual para o controle G. molesta e convencional, em seis datas de avaliação, em pomares de macieira 'Fuji' , Fraiburgo (SC), 1999/2000

\begin{tabular}{llcccccc}
\hline \multirow{2}{*}{ Tratamento } & Praga & \multicolumn{5}{c}{ Data de avaliação } \\
\cline { 3 - 7 } Confusão sexual & B. cranaodes & $12 / 4 / 2000$ & $17 / 3 / 2000$ & $2 / 3 / 2000$ & $15 / 2 / 2000$ & $26 / 1 / 2000$ & $2 / 12 / 1999$ \\
\hline & G. molesta & $0,41 \mathrm{~b}$ & $0,30 \mathrm{a}$ & $0,26 \mathrm{a}$ & $0,46 \mathrm{a}$ & $0,46 \mathrm{a}$ & $0,1 \mathrm{a}$ \\
Convencional & B. cranaodes & $0,44 \mathrm{a}$ & $0,14 \mathrm{a}$ & $0,2 \mathrm{a}$ & $0,2 \mathrm{a}$ & $0,2 \mathrm{a}$ & $0,09 \mathrm{a}$ \\
& G. molesta & $0,02 \mathrm{a}$ & $0,0 \mathrm{a}$ & $0,0 \mathrm{a}$ & $0,0 \mathrm{a}$ & $0,0 \mathrm{a}$ & $0,0 \mathrm{a}$ \\
\hline
\end{tabular}

Médias seguidas de mesma letra para a mesma espécie de inseto nos dois tratamentos não diferem estatisticamente entre si, pelo teste de Tukey $(\mathrm{P}<0,05)$.

\section{CONCLUSÔES}

1. A técnica de confusão sexual utilizando acetato de $(\mathrm{Z} / \mathrm{E})$ - 8 - dodecenila $14 \% \mathrm{p} / \mathrm{v}$ para o controle de G. molesta em macieira é eficiente.

2. O uso de feromônio para controle de G. molesta pode ser utilizado em associação com o manejo de B. cranaodes e A. fraterculus.

\section{REFERÊNCIAS}

ANDREI, E. Compêndio de defensivos agrícolas. São Paulo: Andrei Editora, 1999. 672p.

AQUINO, F.M.; BENITEZ, R.M. Cadeia produtiva da maçã: produção, armazenagem, comercialização, industrialização e financiamento do BRDE na região Sul do Brasil. Porto Alegre: BRDE, 2005. $65 \mathrm{p}$.

BOTTON, M.; KULCHESKI, F.; COLLETTA, V.D.; ARIOLI, C.J. PASTORI, P.L. Avaliação do uso do feromônio de confundimento no controle de Grapholita molesta (Lepidoptera: Tortricidae) em pomares de pessegueiro. Idesia, v. 23, p.43-50, 2005.
BOUVIER, J. C.; BROSSE J.; B. SAUPHANOR, Insecticides. La résistance de Carpocapse. L'Arboriculture fruitière, Montauban, v. 479, p. 21-23, 1995.

CHARMILLOT, P. J., Arboriculteur, prends garde: la carpocapse résistant menace. Revue Suisse de Viticulture, Arboriculture e Horticulture, Nyon, v. 27, p. 71, 1995.

CHARMILLOT, P. J.; PASQUIER D. Petite tordeuse Grapholita lobarzewskii : lutte par la technique de confusion et dynamique des populations. Revue Suisse de Viticulture, Arboriculture e Horticulture, Nyon, v. 33, p. 119-124, 2001.

CHARMILLOT, P. J.; PASQUIER D.; DORSAZ L.; KEIMER CH.; HERMINJARD PH.; OLIVIER R.; ZUBER, M. Lutte par confusion contre carpocapse Cydia pomonella L. en Suisse en 1996 au moyen des diffuseurs Isomate-C Plus. Revue Suisse de Viticulture, Arboriculture e Horticulture, Nyon, v.29, p. 91-96, 1997.

DEGEN, TH.; CHEVALLIER, A.; FISCHER, S. Evolution de la lutte phéromonale contre les vers de la grappe. Revue Suisse de Viticulture, Arboriculture e Horticulture, Nyon, v.37: 273-280, 2005.

GONZALEZ, R.H. Las polillas de la fruta en Chile. Santiago: Universidade de Chile, 2003. 188 p. (Série Ciencias Agronômicas, 9) 
HICKEL, E.R., Pragas do pessegueiro e da ameixeira e seu controle no Estado de Santa Catarina. Florianópolis: Epagri, 1993. 45 p. (Boletim Técnico, 66)

KOVANCI, O. B., WALGENBACH, J. F., KENNEDY, G. G., BORCHERT, D. Evaluation of extended-season mating disruption of the Oriental fruit moth Grapholita molesta (Busck) (Lep., Tortricidae) in apples. Journal of Applied Entomology, Hamburg, v. 128, p. 664-669, 2004.

KOVANCI, O.B.; WALGENBACH, J.E.; KENNEDY, G.G.; SCHAL. J.E.E. Effects of application rate and interval on the efficacy of sprayable pheromone for mating disruption of the Oriental fruit moth Grapholita molesta. Phytoparasitica, Rehovot, p. 33, p. 334-342, 2005.

MOLINARI, F.; GRAVEDI, P.; RAMA, F.; REGGIORI, F.; DALPANE, M.; GALASSI, T. L'uso dei feromoni secondo il método del 'disorientamento' nella difesa des pesco da Cydia molesta e Anarsia lineatella. Atti delle Giornate Fitopatologiche, Bologna, v. 1, p. 341-348, 2000.
MONTEIRO L.B. Manejo integrado de Panonychus ulmi (Koch) (Acari: Tetranychidae) em Macieira. Primeiras experiências com a introdução de Neoseiulus californicus (McGregor) (Acari: Phytoseiidae). In Simpósio Fruticultura de Clima Temperado do Cone Sul, Caçador, SC. Revista Brasileira Fruticultura, Jaboticabal, v.16, p. 46-53, 1994.

ORTH A.I.; RIBEIRO L.G.; REIS, W. Manejo de pragas. In: Manual da cultura da macieira, Florianópolis: EPAGRI, 1986. 562 p.

SAUPHANOR, B.; BOUVIER J.C. Cross-resistance between benzoylureas and benzoylhydrazines in the codling moth, Cydia pomonella L. Journal of Pesticides Sciences, Kyoto, p.45: 369-375, 1995.

VILELA, E.F.; LUCIA T.M.C.D. Feromônios de insetos: Biologia, química e emprego no manejo de pragas. Viçosa: Imprensa Universitária UFV, 2001. 155 p. 